\title{
The impact of four common lumbar spine diagnoses upon overall health status
}

\author{
Michael A. Pahl \\ Thomas Jefferson University and The Rothman Institute
}

Brian Brislin

Thomas Jefferson University Hospital and The Rothman Institute

Scott Boden

The Emory Spine Center

Alan S. Hilibrand

Thomas Jefferson University Hospital and The Rothman Institute

Alexander Vaccaro

Thomas Jefferson University Hospital and The Rothman Institute

Follow this and additional works at: https://jdc.jefferson.edu/orthofp

Part of the Orthopedics Commons

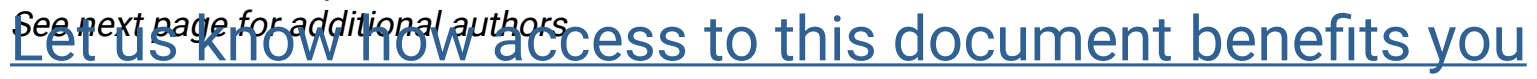

\section{Recommended Citation}

Pahl, Michael A.; Brislin, Brian; Boden, Scott; Hilibrand, Alan S. ; Vaccaro, Alexander; Hanscom, Brett; and Albert, Todd J., "The impact of four common lumbar spine diagnoses upon overall health status" (2006). Department of Orthopaedic Surgery Faculty Papers. Paper 2.

https://jdc.jefferson.edu/orthofp/2

This Article is brought to you for free and open access by the Jefferson Digital Commons. The Jefferson Digital Commons is a service of Thomas Jefferson University's Center for Teaching and Learning (CTL). The Commons is a showcase for Jefferson books and journals, peer-reviewed scholarly publications, unique historical collections from the University archives, and teaching tools. The Jefferson Digital Commons allows researchers and interested readers anywhere in the world to learn about and keep up to date with Jefferson scholarship. This article has been accepted for inclusion in Department of Orthopaedic Surgery Faculty Papers by an authorized administrator of the Jefferson Digital Commons. For more information, please contact: JeffersonDigitalCommons@jefferson.edu. 
Authors

Michael A. Pahl, Brian Brislin, Scott Boden, Alan S. Hilibrand, Alexander Vaccaro, Brett Hanscom, and Todd J. Albert 


\section{The Impact of Four Common Lumbar Spine \\ Diagnoses Upon Overall Health Status}

Michael A. Pahl, MD

Resident, Department of Orthopaedic Surgery

Thomas Jefferson University and the Rothman Institute

Philadelphia, PA 19107

Brian Brislin, MD

Resident, Department of Orthopaedic Surgery

Thomas Jefferson University Hospital and the Rothman Institute

Philadelphia, PA 19107

Scott Boden, MD

The Emory Spine Center

Decatur, GA 30033

Alan S. Hilibrand, MD

Professor, Department of Orthopaedic Surgery

Thomas Jefferson University Hospital and the Rothman Institute

Philadelphia, PA 19107

Alexander Vaccaro, MD

Professor, Department of Orthopaedic Surgery

Thomas Jefferson University Hospital and the Rothman Institute

Philadelphia, PA 19107

Brett Hanscom

The Spine Center at DHMC

One Medical Center Drive

Todd J. Albert, MD

Chairman, Department of Orthopaedic Surgery

Thomas Jefferson University Hospital and the Rothman Institute Philadelphia, PA 19107

Corresponding Author:

Todd J. Albert, MD

925 Chestnut Street, $5^{\text {th }}$ floor

Philadelphia, PA 19107

Phone: (267) 339-3617

Fax: (215) 503-0580

Email: tjsurg@aol.com 


\section{The Impact of Four Common Lumbar Spine Diagnoses Upon Overall Health $\underline{\text { Status }}$}

\section{$\underline{\text { Abstract }}$}

Background Context: The SF-36 health survey has been shown to be a valid instrument when used to measure the self-reported physical and mental health of patients. The impact of lumbar spinal disorders can be assessed as the difference between the SF-36 scale scores and age-andgender specific population norms.

Purpose: To establish the impact upon the self-reported health status of patients with one of four common lumbar spinal diagnoses.

Study Design: A cross-sectional, observational assessment of the health status of spine patients.

Methods: Data from patients presenting to the participating centers of the National Spine Network with low back pain and/or leg pain, was collected prospectively using the Health Status Questionnaire 2.0. A database search identified patients with either herniated nucleus pulposus with radicular pain (HNP), lumbar spinal stenosis without deformity (SPS), degenerative spondylolisthesis (DS), and painful disc degeneration/spondylosis (DDD). The mean SF-36 scale scores were generated for each of the diagnostic groups. The impact of these diagnoses on health status was determined as the calculated difference from the age-and-gender specific population norms for each of the eight health scale scores. These scores, usually negative in this population, represent how far below normal these patients are. The analysis was stratified according to the age of the patients ( $<40 \mathrm{yrs}, 40-60 \mathrm{yrs},>60 \mathrm{yrs}$ ). Analysis of variance and pairwise comparison with Bonferroni correction were used to assess the significance of differences across diagnosis and age groups. 
Results: Data from a total of 4,442 patients was available for this study. All four diagnostic groups had large, negative impact scores for the eight general health scales with the greatest impact upon the three scales that best measure physical health. The greatest impact on these physical health scales (physical functioning, role-physical, and bodily pain) was seen in the HNP diagnostic group. The younger age groups ( $<40$ yrs and $40-60 \mathrm{yrs})$ had the greatest physical impairment when compared to the age-and-gender specific population norms. Analysis of variance showed a significant relationship between diagnosis and SF-36 scores, and between age groups and SF-36 scores.

Conclusions: All four lumbar spine disorders have a significant negative impact on all eight of the SF-36 scales. The greatest negative impact was seen in those scales that measure physical health (RP, PF, and BP). The HNP diagnostic group experienced a significantly greater impact upon these three scales. This diagnostic group had the youngest patients, whose baseline physical functional status would be expected to be the most optimal. When we stratified by age in all the diagnostic groups, the greatest negative impact scores for physical health were seen in the $<40$ yrs and 40-60 yrs age groups. These patients were also more likely to perceive their health as poor, experience decreased energy, and have more social impairment when compared to their age/gender norms. 


\section{$\underline{\text { Key Words }}$}

Spinal disorders; SF-36; health status; negative impact; physical impairment

\section{Key Points}

- The "negative" impact of lumbar spinal disorders can be assessed as the difference between the SF-36 scale scores and the age-and-gender specific population norms.

- All four lumbar spine disorders have a significant "negative" impact on all eight of the SF-36 scales.

- The greatest "negative" impact was seen in those scales that measure physical health (RP, $\mathrm{PF}$, and BP). 


\section{PRECIS}

Low back pain is typically caused by one of four common lumbar spine disorders. All four of these disorders may cause significant functional impairment and even disability. The objective of this study was to establish the impact upon the self-reported health status of patients with one of four common lumbar spinal diagnoses. 


\section{Introduction}

The yearly incidence of low back pain in the United States is between 15-20\% and the lifetime prevalence of low back pain is about $70-80 \%$. It is the most frequent cause of physical limitations in patients below age 45 , the second most common reason for physicians visits, and the third ranking reason for surgical procedures. Approximately $1-2 \%$ of the population of the United States is either chronically or temporarily disabled secondary to back pain. ${ }^{1}$ These disorders have a significant impact on patients in terms of loss of activities and lifestyle, lost wages and productivity, and disability.

While there are numerous causes of low back pain, it is most often attributed to one of four common lumbar spine disorders. Herniated lumbar discs are typically found in younger patients and present with radicular type symptoms in the lower extremities. Spinal stenosis is a disorder more commonly found in elderly patients and is the most common diagnoses in Medicare patients undergoing lumbar spine surgery. ${ }^{2}$ Patients with this disorder may present with back pain, radicular type symptoms, and commonly problems walking secondary to neurogenic claudication. Degenerative spondylolisthesis is a form of segmental instability, which often occurs in conjunction with spinal stenosis. It usually affects patients in their fifth and sixth decades of life and may present with symptoms of neurogenic claudication and radiculopathy. Lumbar disc degeneration and/or collapse have been observed at one or more level in the vast majority of all patients by the age of 50 , and may begin as early as age $20 .^{3}$ These patients usually present with back pain without associated radiculopathy or neurologic dysfunction in the lower extremities, and represent a much greater challenge to the treating surgeon in terms of presentation, clinical imaging, and management than the other three diagnoses. 
All four of these disorders may cause significant functional impairment and even disability in many otherwise healthy patients. Fanuele and Weinstein recently demonstrated that patients with spinal complaints had significantly reduced health status, which was comparable to the impact of many chronic medical diseases. ${ }^{4}$ However little has been written comparing the relative impairment caused by each of these four diagnostic categories. Therefore, the objective of this study was to establish the impact of lumbar disc herniation, disc degeneration, spinal stenosis, and degenerative spondylolithesis upon the self-reported health status of patients based upon direct comparison to age-and-gender specific population norms.

\section{Methods}

This is a study of the patients of the National Spine Network, a consortium of 28 centers focused on the treatment of neck and back problems. The patients were enrolled at their initial visits to the spine clinics of the participating centers. The intake information consisted of a questionnaire concerning demographic information (age, gender, and race), work status, spine symptoms, and the Medical Outcomes Trust's Short Form 36 (SF-36) Health Status Questionnaire. The treating physician completed the intake evaluation by answering questions regarding presenting spine symptoms, diagnostic imaging, medical comorbidities, the specific diagnosis, and finally the treatment plan.

The SF-36 is a well-validated health status survey that has been used in a broad variety of patient populations for its brevity, ability to discriminate among disease states, and acceptability to patients. Eight health scales are measured including: physical functioning (PF), role limitations due to physical symptoms (RP), bodily pain (BP), general health or health perception (HP), energy and fatigue (EF), social functioning (SF), role limitations due to emotional symptoms 
(RE), and mental health $(\mathrm{MH})$. The scales are scored on a range of $0-100$ with a higher score representing better physical function, better health, and better physical and mental health. The scales differ in their ability to measure physical and mental function. The scales are generally presented with the physical measures $(\mathrm{PF}, \mathrm{RP}, \mathrm{BP})$ grouped together and mental measures $(\mathrm{MH}$, $\mathrm{RE}$ ) grouped together. Health perception, energy and fatigue, and social functioning scales tend to fall in between, and have been shown to correlate with both physical and mental health. ${ }^{5}$

All of the information regarding a patient's initial visit was collected and collated into the large database of patients maintained by the NSN. Four lumbar spine diagnoses, as determined by the treating physicians based on clinical and radiographic evaluation, were chosen for evaluation: herniated nucleolus pulposus with radicular pain (HNP), lumbar spinal stenosis without deformity or spondylolisthesis (SPS), degenerative spondylolisthesis (DS), and painful disc degeneration/spondylosis (DDD). A database search identified the available patients in these diagnostic groups and tabulated the SF-36 scale scores. The impact of these diagnoses on health status was determined as the calculated difference from the age-and-gender specific population norms for each of the eight health scale scores. We call these "impact scores." Additionally, we assessed the intake information and further stratified our analysis of the scale scores and the impact scores according to patient age $(<40$ years, $40-60$ years, $>60$ years). Analysis of variance and pair wise comparisons with Bonferroni correction was used to compare the relationship between impact scores and the four lumbar diagnoses and after being stratified into the three age subgroups.

\section{$\underline{\text { Results }}$}


The NSN database has collected information on 11,029 patients treated at the participating centers for various disorders of the lumbar spine. Data from a total of 4,442 patients with one of the four lumbar diagnoses was available for this observational study. Patients included in the database without one of the four diagnoses were excluded. There were 1,908 patients with a diagnosis of herniated nucleolus pulposus with radicular pain, 1,313 patients in the spinal stenosis diagnosis group, 182 with a degenerative spondylolisthesis, and 1,039 patients in the degenerative disc disease diagnosis group. The age among patients with HNP was $43.7 \pm 12$ years, with SPS was $64.9 \pm 12$, with DS was 56.815 , and with DDD was $49.0 \pm 15$. There were 2039 female patients and 2403 male patients.

The mean scores for each of the SF-36 scales are illustrated by the diagnostic groups in Figure 1. While some of the differences in scores between diagnoses were small (0.7 to 13.4), all were significant ( $\mathrm{p}<0.0001$ to 0.0006$)$. These impact scores are presented in Figure 2. The HNP diagnostic group demonstrated significantly larger impact scores for seven of the eight scales ( $p<0.0001$ to 0.0028 , HP not significant). The three largest differences were seen in the RP, PF, and BP scales, which represent greater physical impairment and limitations and increased pain in these patients.

The diagnostic groups were stratified according to age. For all of the diagnostic groups, there were only minor differences in the scale scores among the three age groups ( $<40$ yrs, $40-60$ yrs., $>60 \mathrm{yrs})$ (not presented). However, with respect to the impact scores younger patients ( $<40 \mathrm{yrs}$ and 40-60yrs) in all four diagnostic groups experienced a significantly greater impact upon their physical health scales (PF, RP, BP) versus the older $(>60)$ age group $(\mathrm{p}<0.0001$ to 0.0023$)$ 
(Figure 3). Younger patients in the four diagnostic groups also had a significant negative impact on their perception of their health, less energy and greater fatigue, and greater social impairment ( $p<0.0001$ to 0.05$)$. Overall, there were no significant variations in the Mental Health and Role limitations due to emotional symptoms scale scores or differences from age/gender norms.

The fact that HNP patients have the most severe impact scores is primarily due to the fact that HNP patients are younger. Because younger patients had larger impact scores, the higher prevalence of young patients in the HNP group enhanced the overall impact. An additional analysis of variance compared the impact scores across diagnosis groups within each age group. This analysis revealed that patients in the same age group tended to have similar impact scores, regardless of their diagnosis. One exception were the DDD patients, who tended to show less severe impact scores than the other diagnosis groups in all age categories. This finding, however, could be due to the vague nature of painful disc degeneration.

\section{$\underline{\text { Discussion }}$}

Lumbar spine disorders have a significant detrimental impact upon patients' overall health status. $^{4}$ (Figure 2) The results of this study demonstrated this negative impact on all eight of the SF-36 scales for all of the four diagnostic groups. The greatest impact was seen in those scales that measure physical health (RP, PF, and BP) and the HNP diagnostic group experienced a significantly greater impact upon these three scales. This diagnostic group had the youngest patients, whose baseline physical health status would be expected to be the most optimal. When we stratified by age in all the diagnostic groups, the greater negative impact scores for physical 
health were seen in the $<40$ yrs and 40-60 yrs age groups. These patients were also more likely to perceive their health as poor, experience decreased energy, and have more social impairment when compared to their age/gender norms.

This relationship was similar among all four diagnostic groups, although the effect was most pronounced among the HNP patients under 40. These observations differ from a study by Jones et al. who compared pain, functional (Western Ontario and McMaster Universities (WOMAC) Osteoarthritis Index), and health-related quality-of-life (SF-36) outcome after total hip and knee arthroplasty in an older (>80 yrs) and younger population (55-79 yrs). These authors found no age-related differences in the preoperative SF-36 subscores when the scores were compared to age/gender norms. ${ }^{6}$

The greater impact on physical health and functional status is consistent with previous studies identifying patients with a lumbar spine disorder. These studies' results are compared to our present data in Figure 4. In a prospective study of 41 patients (mean age $=45.5 \mathrm{yrs}$ ) before and after lumbar laminectomy for recalcitrant radiculopathy that was resistant to conservative therapy (35 patients with herniated lumbar disc) by Albert, the preoperative SF-36 scale scores most affected in these patients were also the RP, BP, and PF subscores. ${ }^{7}$ Fanuele et al, who quantified the baseline physical functional status for the entire NSN patient database (of which this study population represents a specific subset), also observed significant physical impairment in patients with lumbar spine disorders. ${ }^{4}$ 
The SF-36 has also been used to study a diverse range of orthopedic and non-orthopedic disorders. Figure 5 compares the means scores for patients prior to orthopedic interventions for hip, knee, and shoulder disorders. The scale scores shown for the hip and knee patients represent the preoperative SF-36 profiles for patients undergoing total joint replacement of either the hip or knee disorder such as osteoarthritis. ${ }^{8}$ Rozencwaig assembled similar data for patients with glenohumeral degenerative joint disease of sufficient severity to meet the criteria for shoulder arthroplasty. ${ }^{9}$ As demonstrated in Figure 5, in comparison to the results of the present study show that patients with lumbar spinal disorders experienced greater physical limitations and bodily pain, had less energy and more fatigue, and also had poorer mental health (SF, MH). These comparisons are limited by the absence of age-adjusted normative data for the other orthopaedic conditions.

Figure 6 is a comparison of these four lumbar diagnostic groups' health status with those of patients with AIDS, Cancer, and Depression. ${ }^{10,11}$ While it appears from this comparison that the patients with a lumbar spine disorder included in this study show better mental health (MH) and may perceive better health (HP), the lumbar spine patients have much worse physical health status (RP, PF, BP) including more physical limitations and more bodily pain. These observed differences are consistent with those of Fanuele et $\mathrm{al}^{4}$, and are extremely important when assessing the overall value of prompt and successful treatment of lumbar disorders when compared to other types of medical disease treatments.

There are several limitations to this study. Statistical significance was attributed to small numeric differences between the individual subscores due to the large patient population and the 
clinical relevance of these statistically significant differences is not clear. The authors also acknowledge that since the patients were in many cases seen at tertiary referral practices, the patients enrolled potentially have a greater pathology then patients seen by most physicians; however, a wide cross section of patients were enrolled by both operative and non-operative spine physicians, therefore this should serve as an equalizer in comparing them to the existing norm. In addition, this study did not correlate the negative impact on health status with the need for medications nor the amount of lost work or productivity. The data presented in this study establishes the negative impact upon patients' self-reported health status. This represented their preoperative health status. Further stratification according to the demographic variable of age showed significantly greater impairment in younger patients. The authors believe that these observations and formulation of the "negative impact" score will be useful in future studies that compare diagnosis, demography and patient's self-reported health status.

This study demonstrated impairment of overall health status for all four lumbar spinal disorders studied. Additionally, statistically the greatest impact was seen in the lumbar disc herniation group and among younger patients ( $<60$ years of age). These findings imply that younger patients, especially those with a lumbar disc herniation, are exceptionally limited by these disorders. 


\section{Bibliography}

1. Andersson GBJ. The epidemiology of spinal disorders. In: Frymoyer JW ed. The Adult Spine: Principles and Practice. $2^{\text {nd }}$ ed. Philadelphia: Lippincott-Raven, 1997: 93-141.

2. Atlas SJ, Deyo RA, Keller RB, Chapin AM, Patrick DL, Long JM, Singer DE. The Maine Lumbar Spine Study, Part III: 1-year outcomes of surgical and nonsurgical management of lumbar spinal stenosis. Spine 1996; 21(15): 1787-94.

3. Boden SD, Davis DO, Dina TS, Patronas NJ, Wiesel SW. Abnormal magnetic-resonance scans of the lumbar spine in asymptomatic subjects. A prospective investigation. J Bone Joint Surg Am. 1990; 72 (3): 403-8.

4. Fanuele JC, Birkmeyer NJ, Abdu WA, Tosteson TD, Weinstein JN. The Impact of spinal problems on the health status of patients: Have we underestimated the effect? Spine 2000; 25(12): 1509-14.

5. Ware JE. SF-36 health survey update. Spine 2000; 25(24): 3130-39.

6. Jones CA, Voaklander DC, Johnston DWC, Suarez-Almazor ME. The effect of age on pain, function, and quality of life after total hip and knee arthroplasty. Arch Intern Med 2001; 161(3): 454-460.

7. Albert TJ, Mesa JJ, Eng K, McIntosh TC, Balderston RA. Health outcome assessment before and after lumbar laminectomy for radiculopathy. Spine 1996, 21(8): 960-63.

8. Hozack WJ, Rothman RH, Albert TJ, Balderston RA, Eng K. Relationship of total hip arthroplasty to other orthopaedic procedures. Clin Ortho Rel Res 1997; 344: 88-93.

9. Rozencwaig R, van Noort A, Moskal M, Smith KL, Sidles JA, Matsen FA. The correlation of comorbidity with function of the shoulder and health status of patients who have glenohumeral degenerative joint disease. JBJS [A] 1998; 80-A(8): 1146-53. 
10. Anderson JP, Kaplan RM, Coons SJ, Schneiderman LJ. Comparison of the quality of well being and the SF-36 results between two samples of ill adults: AIDS and other illnesses. J Clin Epidemiol 1998, 51(9): 755-62.

11. Coulehan JL, Schulberg HC, Block MR, Madonia MJ, Rodriquz E. Treating depressed primary care patients improves their physical, mental, and social functioning. Arch Intern Med 1997; 157(10): 1113-20. 